\title{
The Optimal Design Method and Standardized Mathematical Model of Tooth Profile Modification of Spur Gear
}

\author{
Wenjie Mei, ${ }^{1}$ Jingzhou Na, ${ }^{2}$ Fan Yang, ${ }^{3}$ Guike Shen, ${ }^{1}$ and Jiawei Chen ${ }^{4,5}$ \\ ${ }^{1}$ College of Engineering and Technology, Southwest University, Chongqing 400715, China \\ ${ }^{2}$ School of Mathematical Sciences, Capital Normal University, Beijing 100048, China \\ ${ }^{3}$ Institute of Semiconductors, Chinese Academy of Sciences, Beijing 100083, China \\ ${ }^{4}$ School of Mathematics and Statistics, Southwest University, Chongqing 400715, China \\ ${ }^{5}$ College of Computer Science, Chongqing University, Chongqing 400044, China
}

Correspondence should be addressed to Wenjie Mei; xnmwj703@hotmail.com

Received 4 August 2015; Revised 25 November 2015; Accepted 30 November 2015

Academic Editor: Paolo Lonetti

Copyright ( 2016 Wenjie Mei et al. This is an open access article distributed under the Creative Commons Attribution License, which permits unrestricted use, distribution, and reproduction in any medium, provided the original work is properly cited.

The paper reports a tooth profile modification method of spur gear. After establishing a standardized mathematical model for optimized tooth profile and simulating meshing process with ANSYS finite element analysis, we obtained 625 groups of gear models with different modification parameters. The group with minimum transmission errors owns the optimal parameters. Genetic algorithm was adopted in the entire process for the purpose of reducing the variation of transmission errors in meshing process. The arc and parabolic modification were doing the same processing. After comparing the transmission errors fluctuation produced by the meshing process of gear of nonmodification with arc modification and parabolic modification, we found that the best modification effects of arc modification and parabolic modification were both reduced by $90 \%$. The modification method makes the gear drive process more stable and efficient, and it is also promising in general application for gear drive.

\section{Introduction}

As a widely used mode of transmission, gear transmission has been deeply studied. Profile modification means the change of the shape with standard involute curve, and it is a method which is able to improve the performance of gear. As an optimal design of gear transmission, profile modification can significantly reduce the vibration and noise of gears. In order to attain more ideal modification method, researchers have done much work in selection of modification curves, gear parameters, modification effect, and simulation analysis methods.

There are some studies focusing on reducing transmission errors and improving performance of gear drive. Litvin et al. [1] investigate the effect of misalignment and two main functions of transmission errors. It is shown that parabolic function of transmission errors is able to absorb the linear function of transmission errors, and it also studies the vibration of gear drives. Modifying the involute straight tooth spur gear with straight line, parabola and sine curves can improve the dynamic performance of gear drive [2]. New types of facegear have been proposed and researched in transmission by finite element method, and the modified geometry is used to design the face-gear [3]. Özel [4] investigates the radial modification method of using three-dimensional CNC milling tools and determines the parameters of gear transmission errors by combining mathematical formulas.

Some authors investigate many methods to modify the gear to gain better performance of gear. Spitas et al. [5] introduce an idea on using circle curve to replace standard trochoidal root fillet with BEM. The analysis shows that the new teeth have higher bending strength. Kapelevich [6] introduces a novel design of gears with asymmetric teeth which can increase performance of gears, such as the improvement of load capacity and the decrease of vibration level. Zhe et al. [7] chose straight-line and parabolic curve to modify the spur gear. In order to reduce the transmission errors of gear, they did the optimization design of high precision for 
the modification parameter by analyzing the meshing process of spur gear. Few standardized mathematical models of profile modification have been established and studies of profile modification still remain on specific calculation. Through the establishment of mathematical model, modification models can be expressed by mathematical expressions, which are conducive for the realization of universalization and parameterization of modification. This provides a reliable and referential method for the design of profile modification. Furthermore, this paper investigates the modifying effects between arc curve and parabola.

In this paper, we made a contrastive study on the abovementioned modifying effects through ANSYS simulation analysis. Zhongming et al. [8] introduce modeling method of spur bevel gear and then confirm the amount of tooth profile modification according to the analysis of simulation results; Barone et al. [9] evaluate the effect of misalignment and modify the profile of face-gear by finite element simulation. Sankar and Nataraj [10] suggest a method to simulate the modification effect of spur gear by using the ANSYS software, which can work out the conclusion that profile modification is in a position to improve the stress strength of spur gear. As the above finite element analysis only simulates the transmission process, this paper embedded finite element analysis with genetic algorithm for the purpose of reducing the fluctuation of transmission errors.

By building parameterization mathematical model and selecting modification parameters with regularity, we attained the modification curves which correspond to all parameters. After changing the parameters of modification curve in the parameterized mathematical model, an ANSYS finite element simulation analysis was conducted on the parabolic profile that had been modified in different ways. The final data was obtained by using genetic algorithm to conduct several simulated analyses. Optimal modification and modification parameters were determined through data processing.

By selecting the group with minimum transmission errors fluctuation, we obtained the optimal modification quantity and parameters. The arc and parabolic modification were doing the same processing. Comparing the nonmodification with arc and parabolic modification, we found that the best modification effects of arc modification and parabolic modification were both reduced by $90 \%$. This proves that arc and parabolic modification can improve the gear transmission performance and reduce the transmission errors fluctuation markedly.

\section{The Fluctuation Model of Transmission Error}

The purpose of the research is to reduce the degree of fluctuations of transmission error in the process of gear transmission. The following basic model and extensive model of transmission error were used in this paper.

2.1. Basic Model of Transmission Error. In the meshing process, theoretically, when the drive wheel rotates over $\theta_{1}$, the driven wheel rotates over $\theta_{2}$. Because of the elastic deformation, the driven wheel actually rotates over $\theta_{2}^{\prime}\left(\theta_{1}, \theta_{2}\right.$, and $\theta_{2}^{\prime}$ indicate specific angles).

Transmission error is used to describe the instability of meshing gear [11]. TE for displacement in mesh line represents transmission error; then

$$
\mathrm{TE}=r_{b 2}\left(\theta_{2}^{\prime}-\theta_{2}\right),
$$

where $r_{b 2}$ is the radius of indexing circle of driven wheel and transmission error at any meshing point $i$ is $\mathrm{TE}_{i}$. At this point, sum of modification is $e_{i}$ (including tooth profile and tooth pitch deviation) and integrated deformation is $\delta_{i}$ (the deformation quantity of engagement teeth in the direction of the meshing line):

$$
\mathrm{TE}_{i}=e_{i}-\delta_{i}
$$

Transmission error variation obtained from the rotation is $\Delta \mathrm{TE}_{i}$. The gear transmission error variation is defined $\Delta \mathrm{TE}_{i}=\mathrm{TE}_{i}-\overline{\mathrm{TE}}$ and $\overline{\mathrm{TE}}$ is the average value of transmission error variation at all points. When $\Delta \mathrm{TE}_{i}$ is controlled in a smaller range, the purpose of modification can be achieved.

$$
\begin{aligned}
& \text { 2.2. Extended Model of Transmission Error. Consider } \\
& \qquad \begin{array}{l}
e_{i}=E_{f 1}+E_{f 2}+\mathrm{ES}_{A B}, \\
\delta_{i}=\mathrm{DB}_{1}+\mathrm{DB}_{2}+\mathrm{DH}_{1}+\mathrm{DH}_{2}=F_{A} \delta_{A} .
\end{array}
\end{aligned}
$$

$E_{f 1}, E_{f 2}$ are tooth profile deviation of the driving wheel and driven wheel at the meshing point (including modification). $\mathrm{ES}_{A B}$ is the overall pitch deviation for the current meshing tooth and the last meshing tooth; $\mathrm{DB}_{1}, \mathrm{DB}_{2}$ are the bending deflection of the two teeth along the path of contact at the meshing point; $\mathrm{DH}_{1}, \mathrm{DH}_{2}$ are the contact deflection of the two teeth along the path of contact at meshing point; $F_{A}$ is the normal load between the two teeth; $\delta_{A}$ is the deflection under the effect of unit load along the path of contact. The expression is

$$
\begin{aligned}
\mathrm{TE}= & E_{f 1}+E_{f 2}+\mathrm{ES}_{A B} \\
& -\left(\mathrm{DB}_{1}+\mathrm{DB}_{2}+\mathrm{DH}_{1}+\mathrm{DH}_{2}\right) .
\end{aligned}
$$

2.3. Target Model. Modified profile model can be obtained through modification. A finite element simulation analysis was conducted on the modified optimal gear. In the simulation of transmission process, transmission error at meshing point $i$ is $\mathrm{TE}_{i}$, and transmission error variation at every meshing point is $\Delta \mathrm{TE}_{i}$. Data variance of transmission errors was used to express fluctuation degree. Analogizing fitness in biology, we selected $f=n / \sum_{i=1}^{n}\left(\mathrm{TE}_{i}-\overline{\mathrm{TE}}\right)^{2}$, which is called transmission errors fluctuation. Then the larger $f$ is, the smaller the fluctuation of transmission errors would be. $f_{\max }$ is the minimum transmission errors of the gear during meshing and the profile modification has the best optimizing effect.

\section{The Establishment of Different Modification Curves of Involute Tooth Profile}

As shown in Figure 1, $A$ and $B$ are two points on the curve and the coordinates are $\left(x_{A}, y_{A}\right),\left(x_{B}, y_{B}\right)$. The straight-line 
modification has been proved to have the worst modification effect among several modification methods in many researches $[1,2,4]$; thus the straight-line modification is not described here.

3.1. Parabolic Modification. Involute tooth profile modification is optimized by using parabolic curve. Assuming the parabolic curve equation in rectangular coordinate system is $y=a x^{2}+b x+c$; as is shown in Figure 1, from the character of the involute it can be known that involute curve tangent of $B$, $C B$ occurs perpendicular line $B D$. Because $O D \perp B D$, which ensures the steady of gear meshing process, the slope of the $C B$ is $K_{C B}=K_{\mathrm{OD}}$. Because the point of the involute curve is known and radius of the base circle is $R$, the coordinate of $D$ can be known, and then $K_{\mathrm{OD}}=-\cot \alpha$ :

$$
\begin{aligned}
y_{A} & =a x_{A}^{2}+b x_{A}+c, \\
y_{B} & =a x_{B}^{2}+b x_{B}+c, \\
K_{C B} & =K_{O D}=-\cot \alpha=2 a x_{B}+b .
\end{aligned}
$$

Assuming coordinates of points $A$ and $B$ are known, coefficients of $a, b$, and $c$ can be obtained from Formula Set (5), and the parameters of modification can also be determined. It means that equation of parabola can be obtained which plays a role in modification.

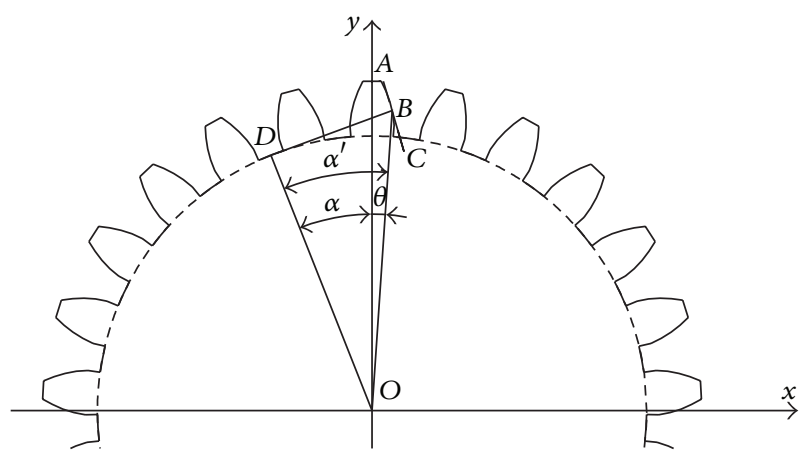

FIGURE 1: Two-dimensional coordinates of gear tooth profile modification.

3.2. Arc Modification. Assuming the arc equation in rectangular coordinate system is $r^{2}=\left(x-x_{0}\right)^{2}+\left(y-y_{0}\right)^{2}$, the arc modification is similar to the parabolic modification as above. From the character of involute it can be seen that the center of the arc is on the generating line of involute. The intersection of the vertical line over the midpoint of $A B$ and involute is the circle center. The equation can be obtained by the above conditions as follows:

$$
\begin{gathered}
y-y_{B}=\tan \alpha \cdot\left(x-x_{B}\right), \\
y-\frac{y_{A}+y_{B}}{2}=\frac{x_{B}-x_{A}}{y_{A}-y_{B}}\left(x-\frac{x_{A}+x_{B}}{2}\right) .
\end{gathered}
$$

Solve the above two formulas simultaneously. The coordinate of the center of arc is

$$
\begin{aligned}
& x_{0}=\frac{y_{B}-\tan \alpha \cdot x_{B}-\left(y_{A}+y_{B}\right) / 2+\left(\left(x_{B}-x_{A}\right) /\left(y_{A}-y_{B}\right)\right) \cdot\left(\left(x_{A}+x_{B}\right) / 2\right)}{\left(x_{B}-x_{A}\right) /\left(y_{A}-y_{B}\right)-\tan \alpha}, \\
& y_{0}=\tan \alpha \cdot x_{0}-\tan \alpha \cdot x_{B}+y_{B} .
\end{aligned}
$$

$\left(x_{0}, y_{0}\right)$ is the coordinate of the center of circle. Both $A$ and $B$ are on the arc curve. If putting $\left(x_{A}, y_{A}\right),\left(x_{B}, y_{B}\right)$ in the coordinate of the center of arc, the arc equation can be obtained which means the parameters of modification are determined.

\section{General Modification Models}

Standard tooth profile modification mathematical models are rarely established; the studies of modification stay in the stage of specific calculation. This mathematical model can be used in several different occasions, and it achieves the generalization of mathematical model. Doing this can reduce workload and avoid repetitive work. By establishing a general model of tooth profile modification, mathematical models can be obtained as follows.
4.1. Parabolic Modifications. In formula (5), the first item subtracts the second one and substitutes the result into the third:

$$
\begin{aligned}
& \left(y_{A}-y_{B}\right)+\cot \alpha \cdot\left(x_{B}-x_{A}\right) \\
& \quad=\left(x_{B}+x_{A}-2\right)\left(x_{B}-x_{A}\right) \cdot a .
\end{aligned}
$$

$\theta+\alpha=\alpha^{\prime}, \alpha^{\prime}$ is the pressure angle and $\theta=\operatorname{tg} \alpha^{\prime}-\alpha^{\prime}$, so the following formula can be obtained:

$$
\alpha=2 \alpha^{\prime}-\operatorname{tg} \alpha^{\prime}
$$

Formula (7) can be changed to

$$
\begin{gathered}
\left(y_{A}-y_{B}\right)+\cot \left(2 \alpha_{B}^{\prime}-\operatorname{tg} \alpha_{B}^{\prime}\right)\left(x_{B}-x_{A}\right) \\
=\left(x_{B}+x_{A}-2\right)\left(x_{B}-x_{A}\right) \cdot a .
\end{gathered}
$$


Tang et al. [12] give the equation of tooth profile:

$$
\begin{aligned}
& x_{B}=R \cdot \cos \alpha_{B}^{\prime}+\frac{\pi}{180} \cdot R \cdot \alpha_{B}^{\prime} \cdot \sin \alpha_{B}^{\prime}, \\
& y_{B}=R \cdot \sin \alpha_{B}^{\prime}-\frac{\pi}{180} \cdot R \cdot \alpha_{B}^{\prime} \cdot \cos \alpha_{B}^{\prime} .
\end{aligned}
$$

The parabolic equation of modification curve can be written as

$$
\begin{aligned}
y= & a x^{2}+\left(-\cot \alpha-2 a x_{B}\right) x+y_{B}-a x_{B}^{2} \\
& +\left(\cot \alpha+2 a x_{B}\right) \cdot x_{B} .
\end{aligned}
$$

It is

$$
\begin{gathered}
y=a x^{2}+\left[\left(-\cot \left(2 \alpha_{B}^{\prime}-\operatorname{tg} \alpha_{B}^{\prime}\right)-2 a x_{B}\right)\right] x+y_{B} \\
-a x_{B}^{2}+\left[\cot \left(2 \alpha_{B}^{\prime}-\operatorname{tg} \alpha_{B}^{\prime}\right)+2 a x_{B}\right], \\
a=\frac{\left(y_{A}-y_{B}\right)+\cot \left(2 \alpha_{B}^{\prime}-\operatorname{tg} \alpha_{B}^{\prime}\right)\left(x_{B}-x_{A}\right)}{\left(x_{B}+x_{A}-2\right)\left(x_{B}-x_{A}\right)} .
\end{gathered}
$$

In the above equation, if the coordinates of $A$ and $B$ are given, then the parametric equation of parabolic modification curve in formula (13) will be determined.

4.2. Arc Modification. The coordinate of arc center from the above parabolic equation which can be obtained according to formula (7) and formula (9) is

$$
\begin{aligned}
& x_{0}=\frac{y_{B}-\tan \left(2 \alpha_{B}^{\prime}-\operatorname{tg} \alpha_{B}^{\prime}\right) \cdot x_{B}-\left(y_{A}+y_{B}\right) / 2+\left(\left(x_{B}-x_{A}\right) /\left(y_{A}-y_{B}\right)\right) \cdot\left(\left(x_{A}+x_{B}\right) / 2\right)}{\left(x_{B}-x_{A}\right) /\left(y_{A}-y_{B}\right)-\tan \left(2 \alpha_{B}^{\prime}-\operatorname{tg} \alpha_{B}^{\prime}\right)}, \\
& y_{0}=\tan \left(2 \alpha_{B}^{\prime}-\operatorname{tg} \alpha_{B}^{\prime}\right) x_{0}-\tan \alpha x_{B}+y_{B} .
\end{aligned}
$$

The radius of arc modification curve is

$$
r=\sqrt{\left(x_{B}-x_{0}\right)^{2}+\left(y_{B}-y_{0}\right)^{2}} .
$$

Similarly, if the coordinates of $A$ and $B$ are given, the center coordinates and radius can be obtained, which means the equation of modification curve can be obtained.

\section{Finite Element Simulation Analysis}

5.1. Solid Modeling. Choosing gear before and after modification as research object. The parameters of gear are as follows: pressure angle of gear is 20; top gap coefficient is 0.25 ; module is 3 ; teeth number of pinion is 30 ; teeth number of gearwheel is 40. According to gear formula, the dividing circle of gearwheel and pinion is 120 and 90, respectively; the dedendum circle of gearwheel and pinion is 112.5 and 82.5, respectively; the addendum circle of gearwheel and pinion is 126 and 96, respectively; the involute standard gear can be attained by using involute module of AUTOCAD, and then we imported the two-dimensional graphs to ANSYS.

According to the parameter equation of the curve above, taking the points of $A$ and $B$ in accordance with certain rules. Point $A$ starts from the intersection of addendum and involute curve. The $A$ points are taken within the range of $2-10 \%$ of the tooth crown length, and the interval is $2 \%$; point $B$ starts from the starting point of the bottom of the tooth, the $B$ points are taken within the range of $5-25 \%$ of the involute curve, and the interval is $5 \%$; the method above is adopted by both drive and driven gears to obtain modification parameters. According to the positions of $A$ and $B$, the modification curve can be attained by using the general modification models. By using the command stream of ANSYS to do modification on the basis of involute standard gear model, 625 groups of nonstandard gear models can be attained. The methods of processing arc and parabolic modifications are the same as this.

Figure 2 shows the change of the gear tooth profile before and after modification. Figure 3 shows the Finite element analysis of meshing gears.

5.2. Mesh Generation. As is shown in Figure 4, select unit type SOLID185 and set a pair of gears as the same material for easier calculation, defining elastic modulus of materials as $2.06 \times 10^{5} \mathrm{MPa}$, friction coefficient of tooth surface MU as 0.05 , Poisson's ratio PRXY as 0.3 , and mesh density as 17850 . The grids of two gears were divided by sweeping command.

5.3. Create Contact Pairs and Boundary Condition. Set up the tooth profile surface of pinion and gearwheel as a contact pair with ANSYS contact guide. Define the tooth profile surface of pinion as contact surface and the tooth profile surface of gearwheel as target surface. Set the contact stiffness factor (FKN) as 1.0; set the maximum amount of penetration which is allowed by Lagrange algorithm as 0.1 . Contact area should be sufficient to describe the contact behavior as needed. Regard GAUSS integral point as default value of contact check point in a face-to-face contact unit of ANSYS, which can produce more accurate results than node integral term of Newton-Cotes/robatto do.

5.4. Loading, Solution, and Postproc. In the meshing process of gear, drive wheel and driven wheel coupled the full degree of freedom of internal cylindrical surface through node. The translational degree of freedom of inner cylindrical surface of the drive gear is constrained. Drive wheel rotates around dead axle, and it has no axial and radial displacement. The moment 


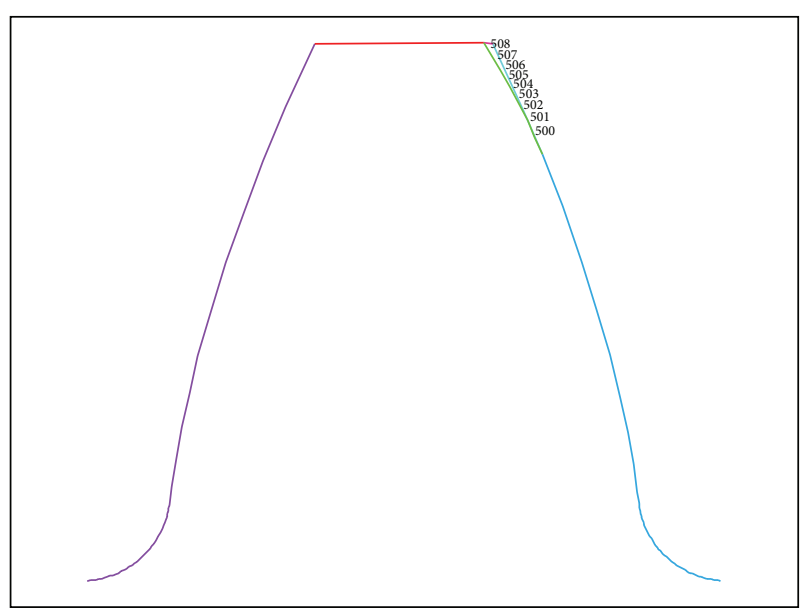

FIGURE 2: Gear profile before and after modification.

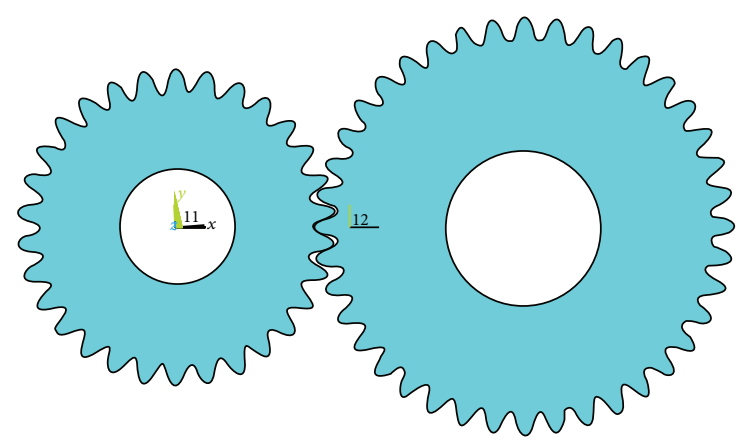

FIGURE 3: Finite element analysis of meshing gears.

of node of drive wheel is exerted as $420000 \mathrm{~N} \cdot \mathrm{m}$; the full degree of freedom of node of driven wheel is constrained.

Considering geometric nonlinearity, each load is divided into 50 substeps, and then the nonlinear iteration is carried out. The multiple cycle solution is executed. Under the condition of satisfying the meshing relationship, the angles of drive and driven wheels are changed slightly each time, and the calculation of deformation of gear under different meshing conditions is repeated.

In the process of Postproc, the angle of drive wheel was output through APDL language. A result is obtained after each cycle calculation. Two kinds of modification methods are, respectively, obtained with the 625 sets of transmission errors, using software to calculate the fluctuation of transmission errors of 625 groups. Finally, the modification parameters, which have optimal results of two kinds of modification methods with minimum transmission errors fluctuation, were obtained; they are the optimal modification parameters.

\section{Application of Genetic Algorithm in Profile Modification}

Proposed by professor Holland and developed by De Jong, genetic algorithm is a simulated algorithm and a method of

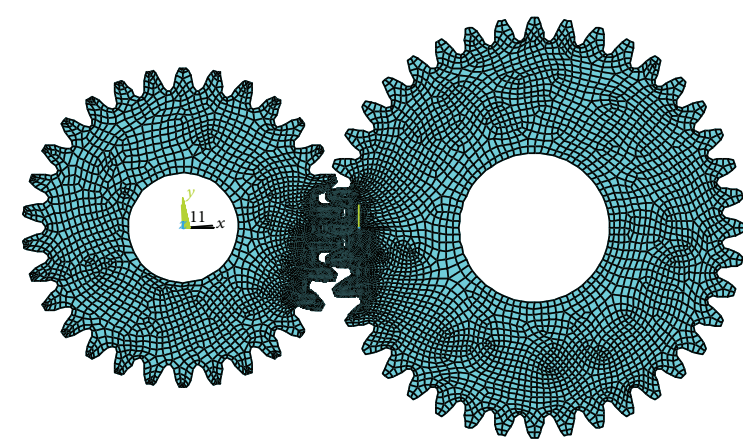

FIGURE 4: Grid figure of gear meshing.

TABLE 1: Two system genes encoding.

\begin{tabular}{lccc}
\hline \multicolumn{2}{c}{ Drive wheel } & \multicolumn{2}{c}{ Driven wheel } \\
$x_{A}$ & $y_{A}$ & $x_{B}$ & $y_{B}$ \\
\hline 011101 & 101011 & 011100 & 111001 \\
\hline
\end{tabular}

random search borrowed from the laws of evolution in biology (mechanism for the survival of the fittest) [13]. Genetic algorithm was used in the entire process. A modification model was established; the process of coordinates $A$ and $B$ was generated according to regularity, which can be seen as genetic algorithms coding; finally, the variation of meshing transmission error of modified gear can be obtained, namely, the digital simulation results of ANSYS, which can be seen as individual fitness, and it reflects the degree of meshing stability. The results of ANSYS can be analogized with the results of ANSYS which can be seen as the largest fitness in biology. According to genetic laws, the objections which have larger fitness are more excellent than others, and thus the individual which has largest fitness is the optimal result. The ideas for the application of genetic algorithm in the entire process are as follows.

Gene algorithms coding of possible corresponding solutions with 6 -digit binary coding. Table 1 shows the gene algorithms coding map of the solution of two parameters $A$ and $B$.

Then we generated the initial groups. The adaptability function is defined as

$$
f=\frac{1}{(1 / n) \sum_{i=1}^{n}\left(\mathrm{TE}_{i}-\overline{\mathrm{TE}}\right)^{2}} .
$$

$\overline{\mathrm{TE}}$ is the average value of TE. Result of decoding is brought into the above function to sequence the chromosomes according to their adaptability values.

After we selected operator, we crossed operator and obtained the Crossover operator. The Mutation operator is obtained by randomly changing the binary code of an allelic gene at mutation probability $P_{m}$.

The process continues unless the groups begin to converge. Otherwise, execute steps above. In the above steps, 
TABLE 2: ANSYS simulation data.

\begin{tabular}{lccc}
\hline $\begin{array}{l}\text { Rotational } \\
\text { angle }\left(^{\circ}\right)\end{array}$ & $\begin{array}{c}\text { Without } \\
\text { modification }\end{array}$ & $\begin{array}{c}\text { Transmission error }\left(\mathrm{TE}_{i}\right) \\
\text { Arc } \\
\text { modification }\end{array}$ & $\begin{array}{c}\text { Parabolic } \\
\text { modification }\end{array}$ \\
\hline 0.00 & 0.5175 & 0.5353 & 0.5360 \\
1.00 & 0.4985 & 0.5364 & 0.5364 \\
2.00 & 0.4840 & 0.5279 & 0.5246 \\
3.00 & 0.4794 & 0.5233 & 0.5203 \\
4.00 & 0.4888 & 0.5263 & 0.5254 \\
5.00 & 0.5094 & 0.5336 & 0.5364 \\
6.00 & 0.5259 & 0.5371 & 0.5331 \\
7.00 & 0.5229 & 0.5291 & 0.5269 \\
8.00 & 0.5215 & 0.5250 & 0.5235 \\
9.00 & 0.5217 & 0.5230 & 0.5222 \\
10.00 & 0.5237 & 0.5245 & 0.5243 \\
11.00 & 0.5274 & 0.5287 & 0.5287 \\
\hline
\end{tabular}

$f=n / \sum_{i=1}^{n}\left(\mathrm{TE}_{i}-\overline{\mathrm{TE}}\right)^{2}$ is the target function $f_{\max }$. The optimized parameter is obtained from calculation of the above function.

\section{Results of Digital Simulation}

(1) As is shown in Table 2, from the finite element simulation, a number of transmission error data can be obtained. We obtained output data of transmission error in three cases, respectively. The data in the following forms are about nonmodification, arc modification, and parabolic modification. With the rotation of the gear, the optimal results of transmission error can be obtained, and the analog gear is $12^{\circ}$ cycles.

Based on the genetic algorithm data from Table 2, the results can be obtained as follows:

without modification

$$
f_{\max }=3498.33
$$

best optimization results of arc modification

$$
f_{\max }=40859.81,
$$

best optimization results of parabolic modification

$$
f_{\max }=31837.41 .
$$

(2) According to Table 2, the transmission error curves can be obtained as follows.

(a) Figure 5 shows the comparison chart of unprocessed simulation data and optimized simulation data of arc modification.

(b) Figure 6 shows the comparison chart of unprocessed simulation data and optimized simulation data of parabolic modification.

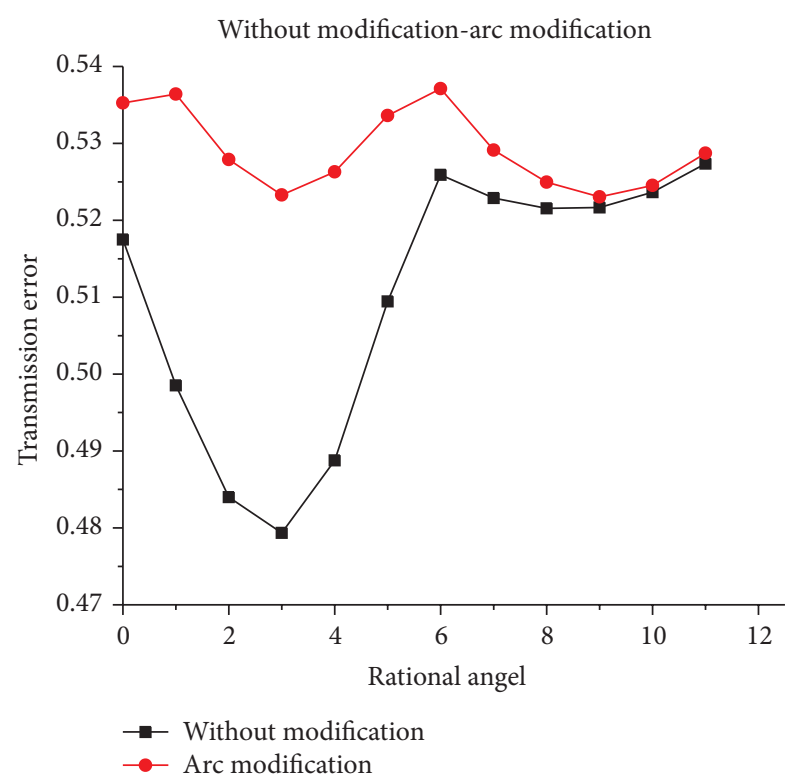

FIGURE 5: Comparison of unprocessed simulation data and optimized simulation data of arc modification.

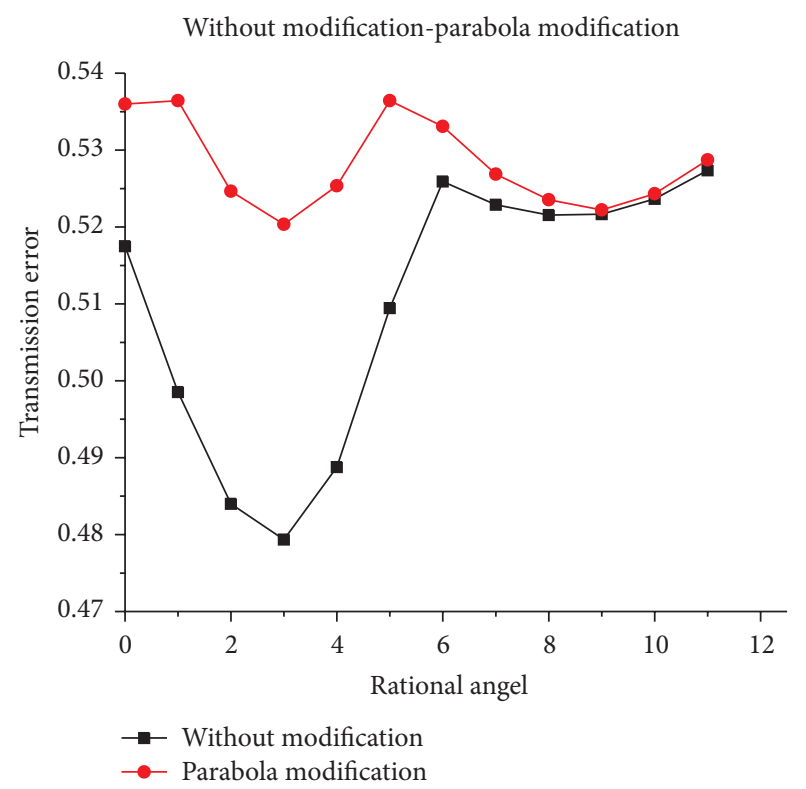

FIgURE 6: Comparison of unprocessed simulation data and optimized simulation data of parabolic modification.

(c) Figure 7 shows the comparison chart of optimized simulation data between arc modification and parabolic modification.

\section{Conclusion}

In this paper, methods and processes of gear tooth profile modification were described by the mathematical model and genetic algorithm. Standardized method of tooth profile modification was established. 


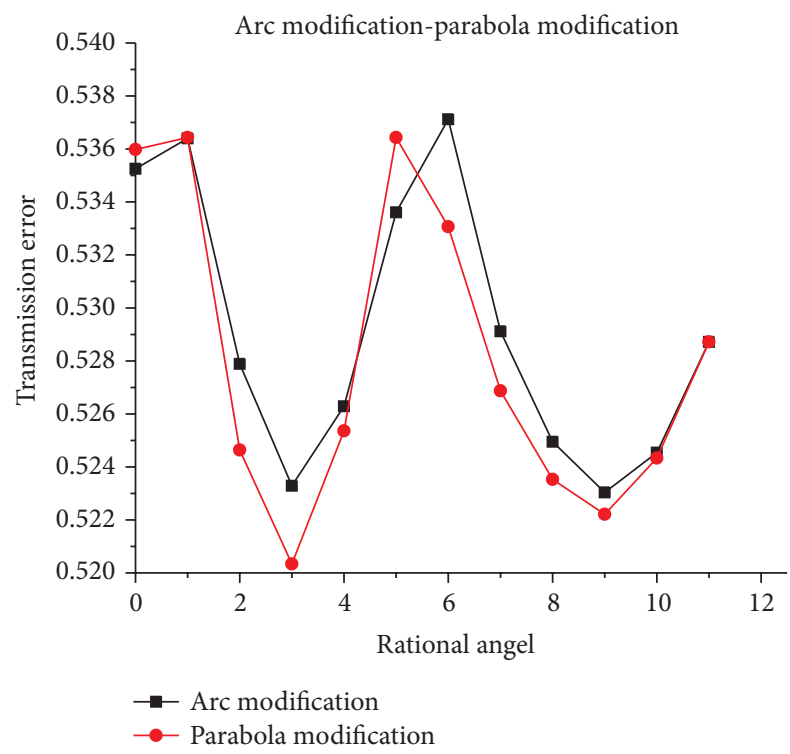

Figure 7: Comparison of optimized simulation data between arc modification and parabolic modification.

Comparing the unprocessed simulation data with the optimized simulation data of arc and parabolic modification, results are obtained as follows.

(1) Parabolic modification and arc modification have played a significant role in the optimization; the best modification effects of arc modification and parabolic modification were both reduced by $90 \%$ compared with nonmodification; $f_{\max }$ of parabolic and arc modification under three cases is much larger than unprocessed $f_{\max }$. Smoothness of the amount of transmission errors of the former two methods is significantly larger than unprocessed one. It means that the transmission errors of gear modification are stable in the process of gear meshing.

(2) $f_{\max }$ of arc modification is similar to that of parabolic modification. It means the optimized effect of the arc modification and the parabolic modification are closely related.

(3) The transmission error without modification is greater than those of both parabolic and arc modification. Compared with parabolic and arc modification, the rotation angle of driven wheel is closer to theoretical value when the drive wheel rotates a certain angle in the condition of nonmodification. Nevertheless, the fluctuation of driven wheel is greater than two types of modifications, which means the transmission error is greater.

\section{Conflict of Interests}

The authors declare that there is no conflict of interests regarding the publication of this paper.

\section{References}

[1] F. L. Litvin, D. Vecchiato, K. Yukishima, A. Fuentes, I. GonzalezPerez, and K. Hayasaka, "Reduction of noise of loaded and unloaded misaligned gear drives," Computer Methods in Applied
Mechanics and Engineering, vol. 195, no. 41-43, pp. 5523-5536, 2006.

[2] C. Zhu, K. Song, and Z. Tian, "Optimum design and experiment study on the gear profile modification," Chinese Journal of Mechanical Engineering, vol. 34, no. 4, pp. 63-68, 1998.

[3] F. L. Litvin, A. Fuentes, and M. Howkins, "Design, generation and TCA of new type of asymmetric face-gear drive with modified geometry," Computer Methods in Applied Mechanics and Engineering, vol. 190, no. 43-44, pp. 5837-5865, 2001.

[4] C. Özel, "A study on cutting errors in the tooth profiles of the spur gears manufactured in CNC milling machine," The International Journal of Advanced Manufacturing Technology, vol. 59, no. 1-4, pp. 243-251, 2012.

[5] V. Spitas, T. Costopoulos, and C. Spitas, "Increasing the strength of standard involute gear teeth with novel circular root fillet design," American Journal of Applied Sciences, vol. 2, no. 6, pp. 1058-1064, 2005.

[6] A. Kapelevich, "Geometry and design of involute spur gears with asymmetric teeth," Mechanism and Machine Theory, vol. 35, no. 1, pp. 117-130, 2000.

[7] Y. Zhe, S. Zhili, and G. Yu, "Optimal design of profile modification curves for spur gears," Journal of Mechanical Transmission, vol. 5, p. 002, 2010.

[8] W. Zhongming, W. Xinyun, X. Juchen, and H. Guoan, "Modeling and dynamic FEA technology of bevel spur based on ANSYS," Journal of Mechanical Transmission, vol. 5, p. 15, 2005.

[9] S. Barone, L. Borgianni, and P. Forte, "Evaluation of the effect of misalignment and profile modification in face gear drive by a finite element meshing simulation," Journal of Mechanical Design, vol. 126, no. 5, pp. 916-924, 2004.

[10] S. Sankar and M. Nataraj, "Profile modification-a design approach for increasing the tooth strength in spur gear," The International Journal of Advanced Manufacturing Technology, vol. 55, no. 1-4, pp. 1-10, 2011.

[11] T. Jinyuan, "Modeling of gear transmission error," Journal of Mechanical Transmission, vol. 6, pp. 13-14, 2008.

[12] J. Y. Tang, C. J. Zhou, and Y. X. Wu, "A method for exact modeling of the bending of spur gear teeth by FEM," Mechanical Science and Technology, vol. 23, no. 10, pp. 1146-1150, 2004.

[13] K. A. De Jong, Analysis of the behavior of a class of genetic adaptive systems [Ph.D. thesis], Department of Computer and Communication Sciences, University of Michigan Ann Arbor, 1975. 


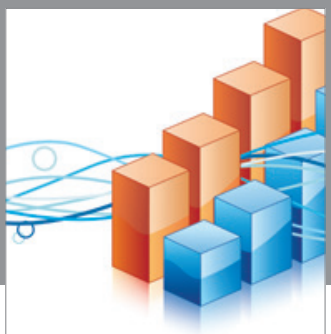

Advances in

Operations Research

vatem alat4

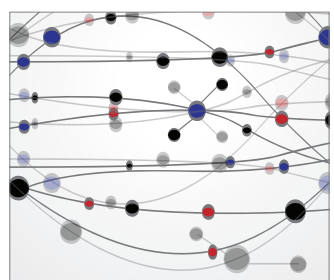

\section{The Scientific} World Journal
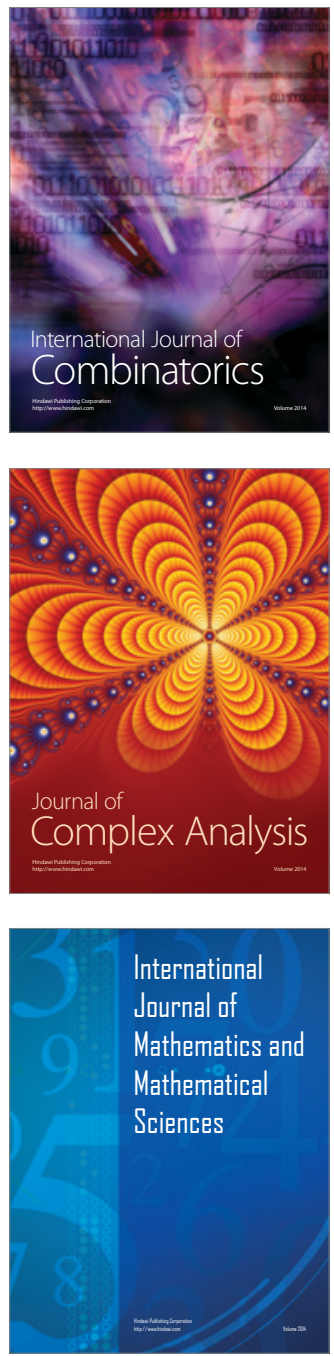
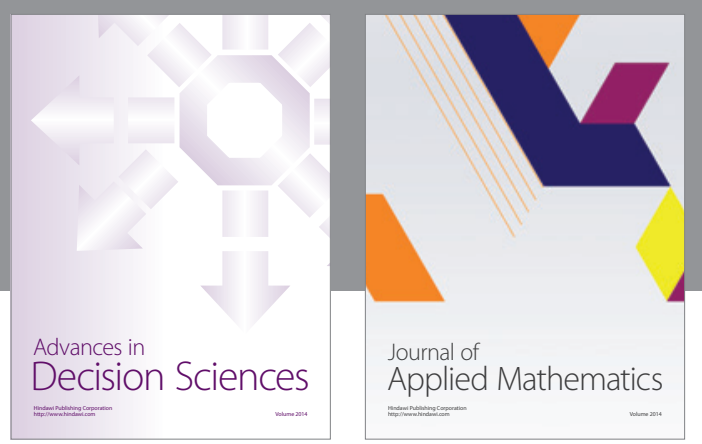

Algebra

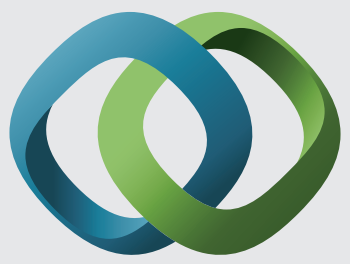

\section{Hindawi}

Submit your manuscripts at

http://www.hindawi.com
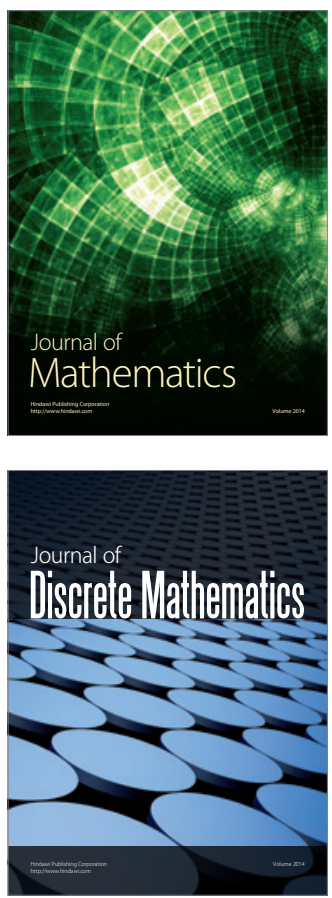

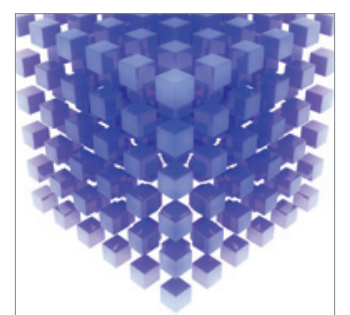

Mathematical Problems in Engineering
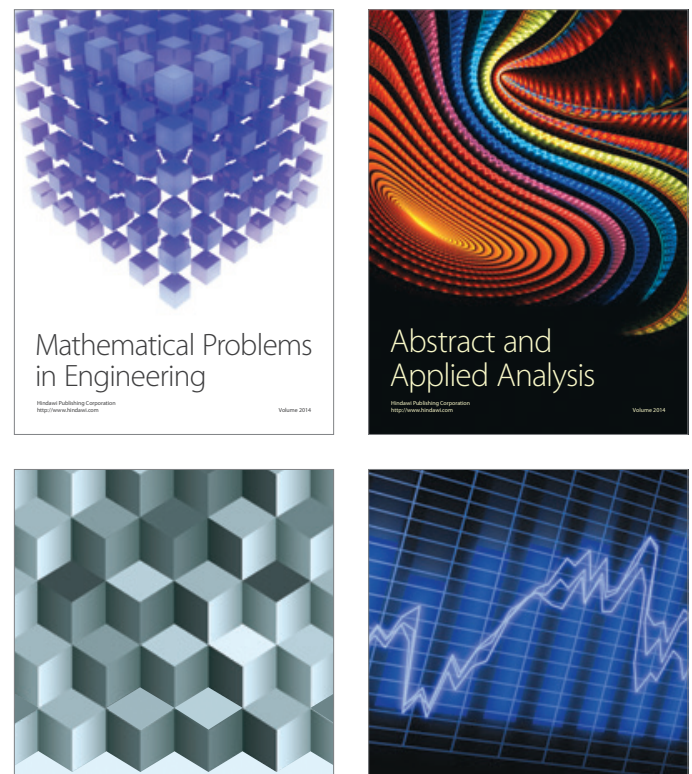

Journal of

Function Spaces

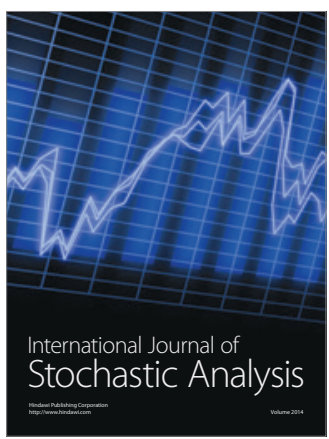

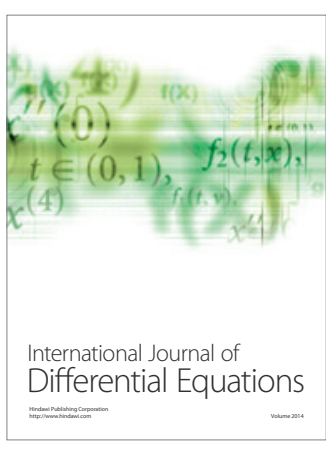
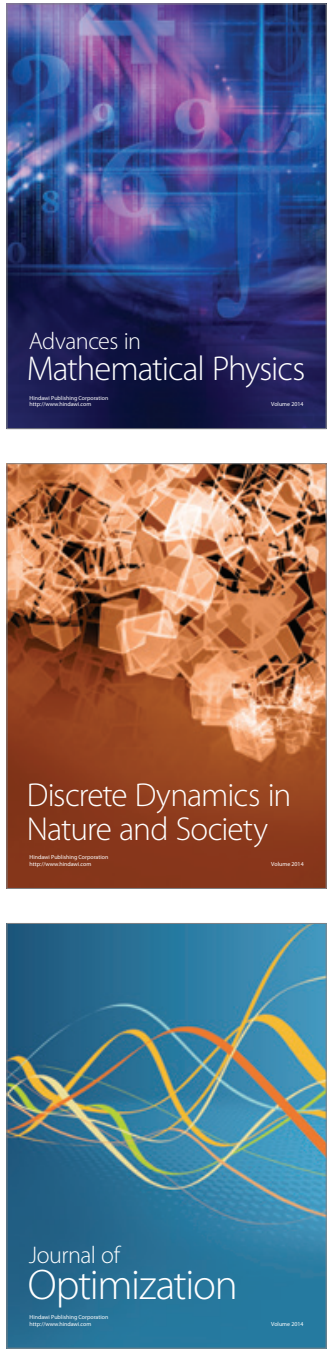